Journal of Information Technology and Digital World (2020)

Vol.02/ No. 01

Pages: 53-63

https://www.irojournals.com/itdw/

DOI: https://doi.org/10.36548/jitdw.2020.1.005

\title{
Service Quality Assurance in Cloud Data Centers Using Migration Scaling
}

\author{
Dr. Akey Sungheetha \\ Data Science SIG member, \\ Computer Science and Engineering, \\ School of Electrical Engineering and Computing, \\ Adama Science and Technology University, \\ Adama, Nazret, Ethiopia. \\ Dr. Rajesh Sharma R \\ Image Processing SIG member, \\ Computer Science and Engineering, \\ School of Electrical Engineering and Computing, \\ Adama Science and Technology University, \\ Adama, Nazret, Ethiopia.
}

\begin{abstract}
The continuous and swift progress in the number of the cloud data centers have led to establishment of multitudes of the computational nodes and the huge paradigm. But the assuring the quality of services through these paradigms is still questionable. So tit has become a prominent areas of research. As the quality of service of the data centers plays a vital role in the user satisfaction. The present work carried out in the paper survey the service quality rendered in the previous similar work, identifies the drawbacks and proposes a strategy of migration taking into consideration the multiple metrics. The proposed structure is validated through the cloud simulator to evince its capability in efficiently handling the resources and guaranteeing the quality of service.
\end{abstract}

Keywords: Cloud Computing, Cloud Data Center, Migration Scaling, Service Quality, Resource Management

\section{Introduction}

The cloud computing's era began few years back from 1960s for the "intergalactic computer network" that was first suggested, nowadays the technology has become popular in a broader range of application and has started to shake-up both the supplier and the IT enterprise. Recently multitudes of companies are adopting to cloud and it has become the lifeblood of the present organizations. It is viewed as the additional stage in the phases of the communication that saves a lot of time and money. The service provided by the cloud enables one to receive a higher quality commodities and knowledge on demand. This has caused many business, organizations and the industries to operate fundamentally from the cloud paving way for productive, flexible and efficient ways of working. So the cloud has become a more prominent for the organization development of digital age.

ISSN: 2582-418X (online) 
Journal of Information Technology and Digital World (2020)

Vol.02/ No. 01

Pages: 53-63

https://www.irojournals.com/itdw/

DOI: https://doi.org/10.36548/jitdw.2020.1.005

The cloud paradigm is available through a well-defined interface, and could be accessed from anywhere. The resources available in the cloud could be scaled up according to the demands of the devices. More over the cloud services are reliable and robust. It capable of offering services on pay as go model, this affords the consumers to have a low capital expenditure.

It integrates the virtualization, on demand deployment, open source software, internet service delivery and data and application. This has caused multiple of organizations to develop dynamic data centers to provide the resources according to the demands. In today's cloud service offerings the providers of cloud are supplying the web based interface and the application programming enabling an organization to use virtualized platforms for storage that are hosted as well as the application environments, delivering services that can incorporated into the own service provisions of the organizations.

The service provisions of the cloud has made it more prominent among a wide range of application and has caused more and more organization to adopt the cloud computing and develop dynamic cloud data centers that are huge, holding lots of computational nodes. But to provide services satisfying the demands, it is mandatory to assure the quality of service by the provider of the service.

Many research work has been published to guarantee the service quality of the multitude of the requirements without taking into consideration the multiple metrics. So the paper put forth the migration mechanism, taking into consideration the multiple metrics to offer assured service quality and perfect management of the resources.

The paper is planned with the section two describing the related works, the section there describing the migration scheme with assured service quality, section four presenting the analysis and the evaluation results and section 5 providing the conclusion.

\section{Related Works}

The related works section present the similar works carried out to deliver the knowledge of the issues related to the assured quality service, and provide the particulars of the metrics that are associated in guaranting the quality of service. Zhang et al [1] proposes the concept to mitigate the power utilization as the results of the high speed links that are very popular nowadays, the proffered method utilizes the policies to regulate the link rate changes in order to have to considerable savings in energy, the prototype to handle the challenges incurred in real time.

ISSN: 2582-418X (online) 
Journal of Information Technology and Digital World (2020)

Vol.02/ No. 01

Pages: 53-63

https://www.irojournals.com/itdw/

DOI: https://doi.org/10.36548/jitdw.2020.1.005

Gunaratne, et al [2] the drastic energy consumption due to the increase of the cloud data centers as a results of swift progress in the cloud adoption made the author to conduct the survey to identify the challenges endured in effectively handling the resources of the cloud Beloglazov, et al [3] to minimize the consumption of energy in turn to reduce the electricity bills and the carbon dioxide foot prints the author discusses the " current taxonomy, energy efficient design, of the computing system covering the hardware, operating system virtualization and data center levels to sort out the energy efficient computing solutions.

Galloway et al [4] a power aware load balancing framework is put forth in the paper utilizing the "PALBA" according to the percentage of utilization. Beloglazov, et al [5] has devised the policies for an energy optimized management of the resources proposing a perfect scheduling algorithm meeting the QOS demands.Huang et al [6] the proposed method organizes an energy aware virtual machine placement to provide a balance between the energy utilization in the sever and the network,

Maurya, et al [7] the author puts forward a method to minimize the number of migrations as well as the energy utilization of the VM migration by incorporating the policies of the minimum migration Graubner, et al [8] devises the "novel strategy for VM consolidation, based on the energy efficiency in storage migration and the live VM migration by utilizing the eucalyptus an open source clone of the AECC" Farooqi, et al [9] elaborates the concepts and the methods utilized to minimize the consumption of the energy and the $\mathrm{Co} 2$ emission by performing a comparative analysis over " green cloud computing."

Piraghaj et al [10] the work focuses on managing the resources of the peas (platform as service) with energy efficiency" Kumar et al [11] conducts the "review on the ubiquitous computing" Smys, S. et al [12], presents the "A Stochastic Mobile Data Traffic Model for Vehicular Ad Hoc Networks"

Bhalaji, N.et al [13] performs the "Delay Diminished Efficient Task Scheduling and Allocation for Heterogeneous Cloud Environment" .Shakya, S et al [14] presents the "An Efficient Security Framework for Data Migration in a Cloud Computing Environment". Karunakaran, V et al [15] puts forward "A Stochastic Development of Cloud Computing Based Task Scheduling Algorithm.". .Bashar, A et al [16]. Proffers the "Secure and Cost Efficient Implementation of the Mobile Computing Using Offloading Technique" Haladu, et al [17] presents the "Optimizing Task Scheduling and Resource allocation in Cloud Data Center using Enhanced Min-Min Algorithm." The figure. 1 below is the basic structure of the clod data centers

Information Technology $\&$ Digital World 
Journal of Information Technology and Digital World (2020)

Vol.02/ No. 01

Pages: 53-63

https://www.irojournals.com/itdw/

DOI: https://doi.org/10.36548/jitdw.2020.1.005

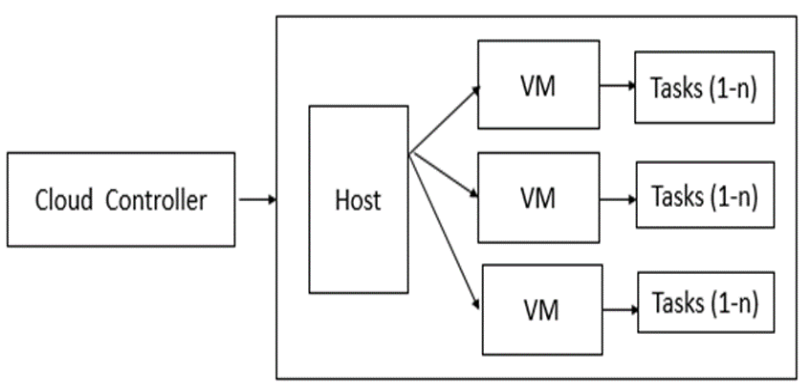

Figure.1 Cloud Data Center

All the above mentioned focuses on any one of the metric to have proper resource management and migration, in most cases the power consumption was considered to be the essential metric in enhancing the QOS and some cases considered the cost and few others the delay. The proposed method considers multiple metric such as the cost, power, delay etc. to have an effective migration with proper resource handling and guaranteed QOS. The figure .2 below shows the classification of the QOS metric in the cloud Data Centers.

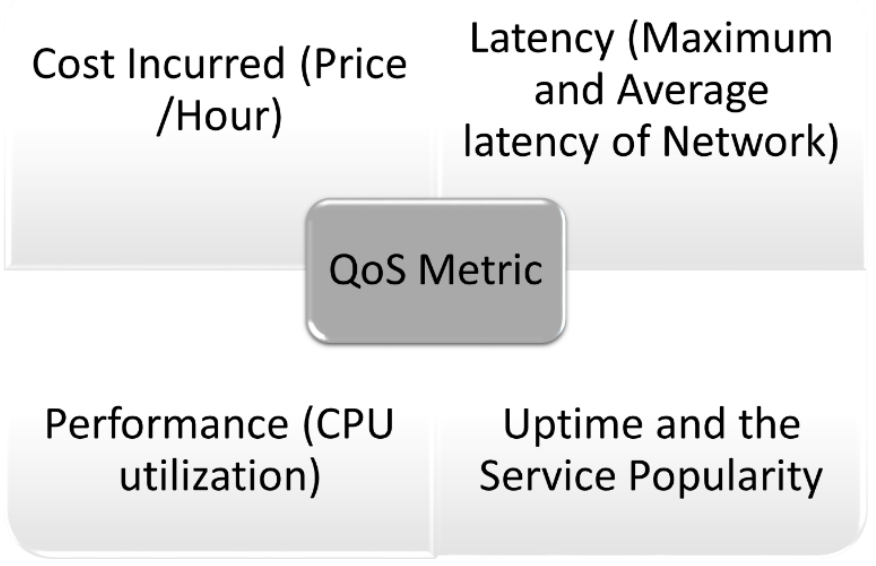

Figure.2 Cloud Data Center QOS Metric

ISSN: 2582-418X (online)

Information Technology

\&

Digital World 
Journal of Information Technology and Digital World (2020)

Vol.02/ No. 01

Pages: 53-63

https://www.irojournals.com/itdw/

DOI: https://doi.org/10.36548/jitdw.2020.1.005

\section{Proposed Work}

The architecture of the proposed scheme is comprised of triple fundamental modules they are the (i) Service Interpreter (Serv-Inprt), Service Tracker (Serv-Track), and the Migration Organizer (Mig-Org). The block diagram in figure .3 depicts the basic strategy of the proposed method.

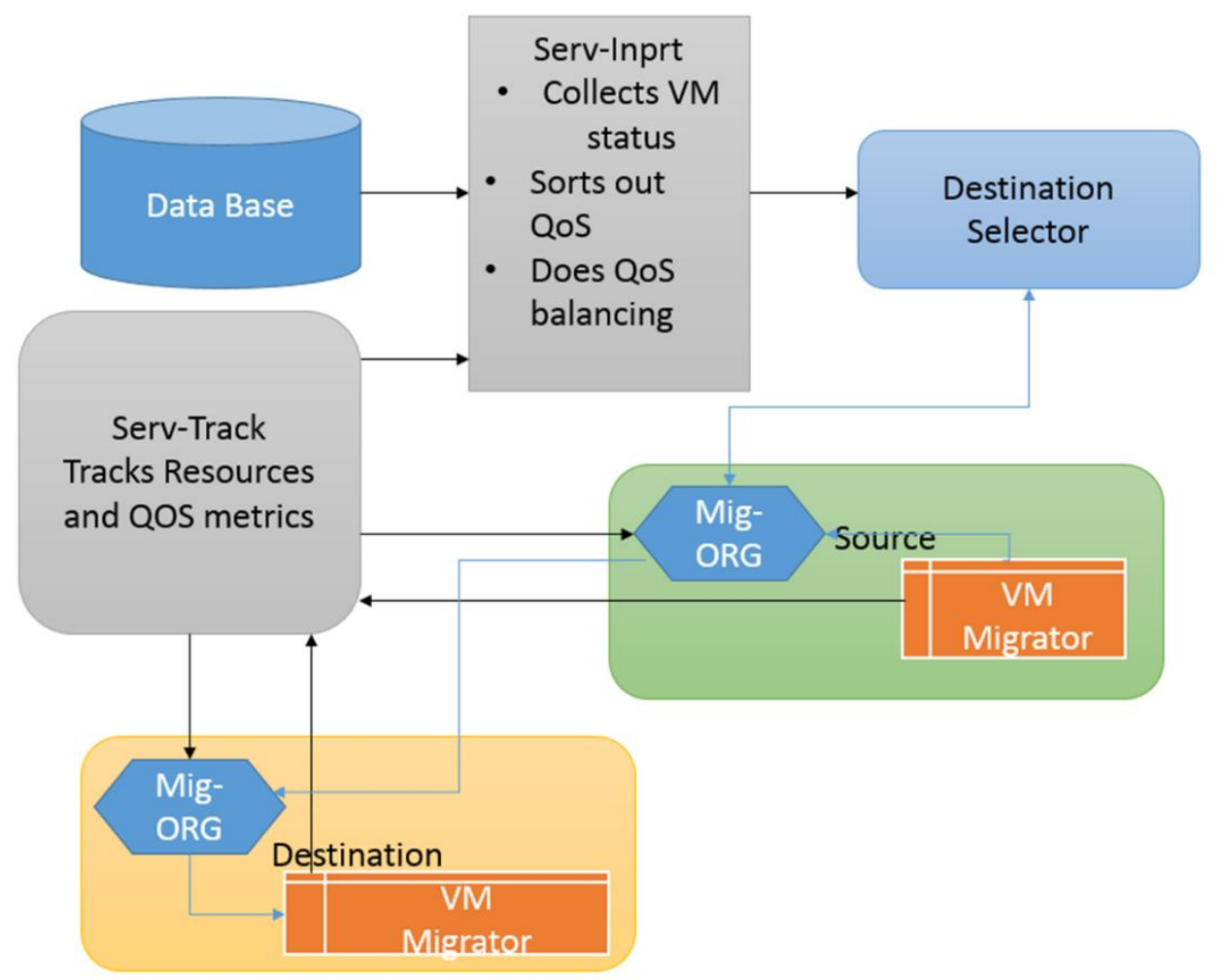

Figure.3 Proposed Strategy

The Serv-Inprt estimates the working position of the VM. This is done as it is very much essential in the process of migration and the opting the destination machine, the Serv-Track keeps the model updated on the information of the machine in the destination according weight and its metric value.

ISSN: 2582-418X (online)

Information Technology

\&

Digital World 
Journal of Information Technology and Digital World (2020)

Vol.02/ No. 01

Pages: 53-63

https://www.irojournals.com/itdw/

DOI: https://doi.org/10.36548/jitdw.2020.1.005

The Serv-Track, tracks the particulars of the resources of the source as well as the machine in the destination along with the utilization of resources and also tracks the information's of the about the configuration of the virtual machine that is very much important in making the decisions about the migration, the information track are forwarded to the Serv-Inprt. Further the Mig-Org is to decide whether the migration has to be done or not taking into consideration the performance metrics. The figure. 4 below is the flow diagram of the proposed strategy.

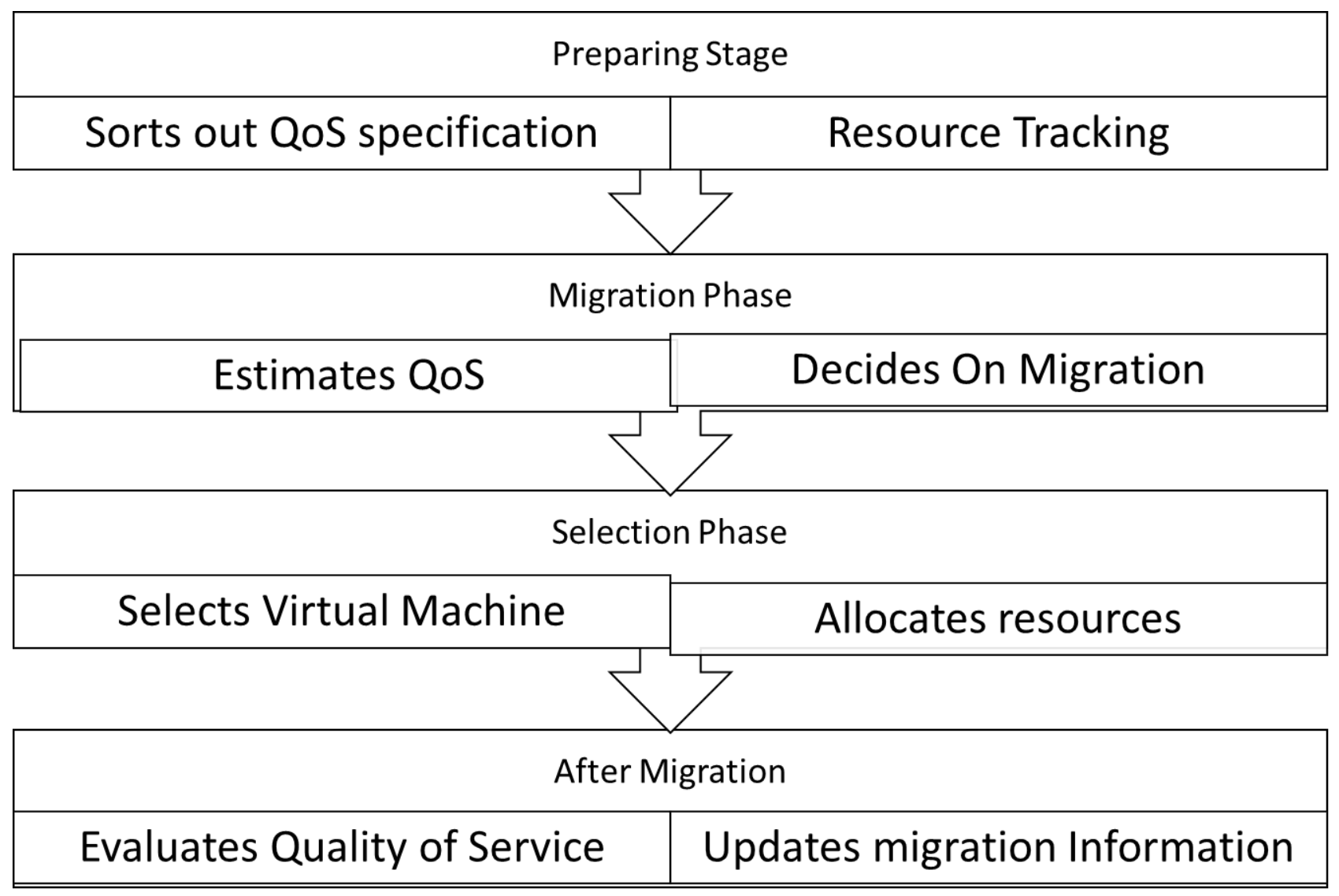

Figure.4 Proposed Flow Diagram

The decision making to maximize the quality of service is done using the priority scheme as shown in equation.1

$$
\text { Decision }_{\text {making }}=\sum_{x} \text { Weight }_{x} M_{x y}
$$


Journal of Information Technology and Digital World (2020)

Vol.02/ No. 01

Pages: 53-63

https://www.irojournals.com/itdw/

DOI: https://doi.org/10.36548/jitdw.2020.1.005

Where the $M_{x y}$ refers to the 'x-th' metric value of ' $y$-th' service

\section{Results Evaluation}

The evaluation of the proposed model is performed using the cloud simulator on the basis of the performance metric such as cost, execution time, response time, latency and the CPU utilization for varying number of loads ranging from 100 to 500 . The proposed strategy provides a more benefits over the conventional static strategies. The Figure. 5 below is the performance of the propose model on terms of the latency, the results observed is compared with the static strategies.

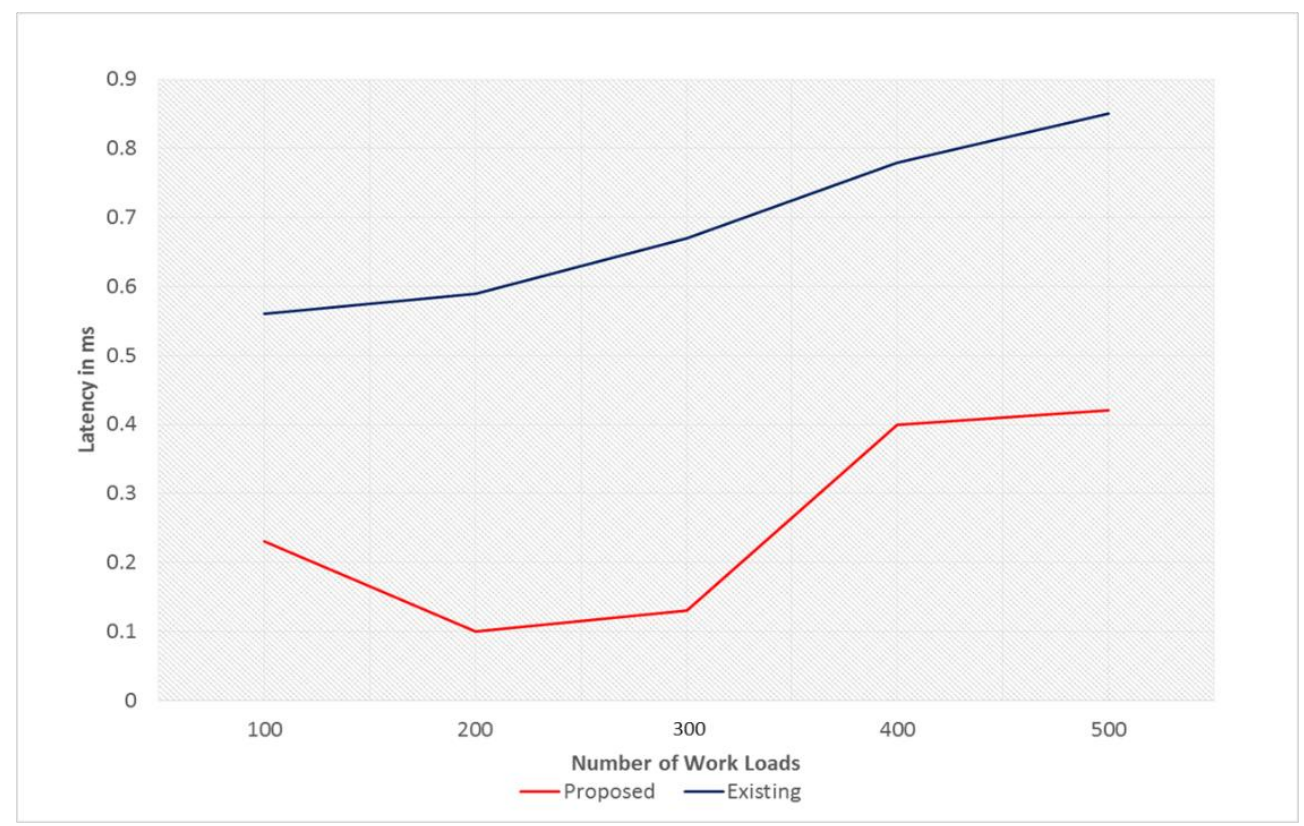

Figure.5 latency

The proposed method affords to deliver a better latency in the completion of the service compared to the Static strategies. The figure. 6 shows the execution time and the response time taken for each task by the proffered model and the conventional method. The average execution time and the response time of the migration strategy put forth is very much less compared to the existing method. 
Journal of Information Technology and Digital World (2020)

Vol.02/ No. 01

Pages: 53-63

https://www.irojournals.com/itdw/

DOI: https://doi.org/10.36548/jitdw.2020.1.005

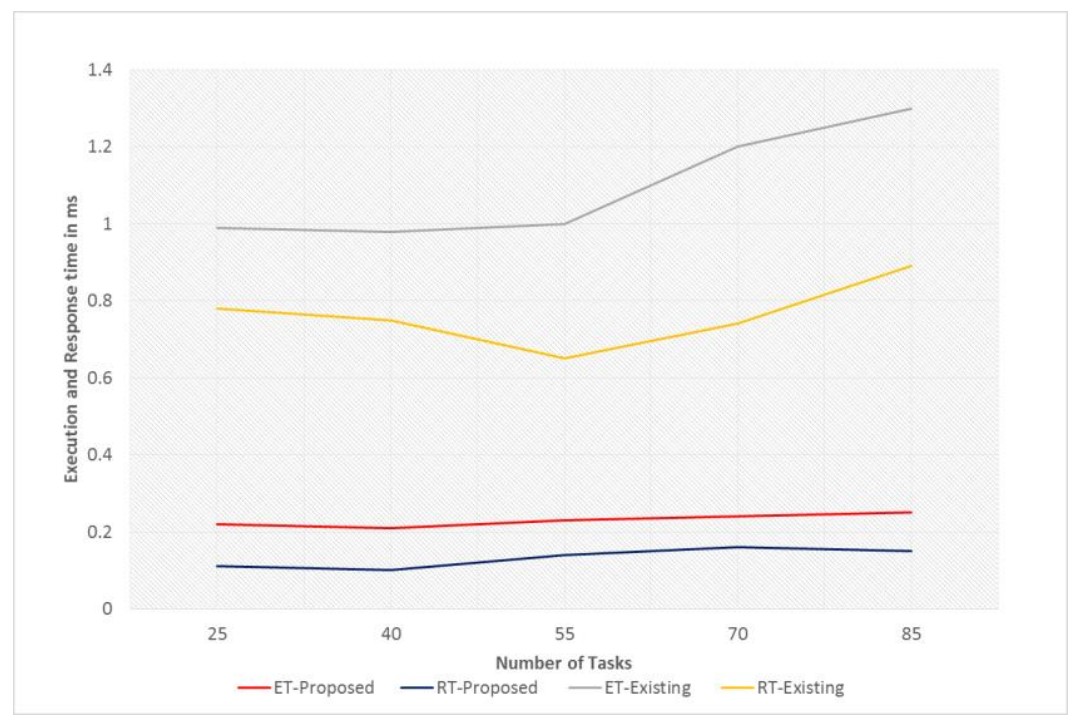

Figure.6 Execution and Response Time

The response below in the figure 7 shows the results observed on the cost incurred and the CPU utilization, the results represented proves the competencies of the method put forth in terms of the CPU utilization and the Cost Spent.

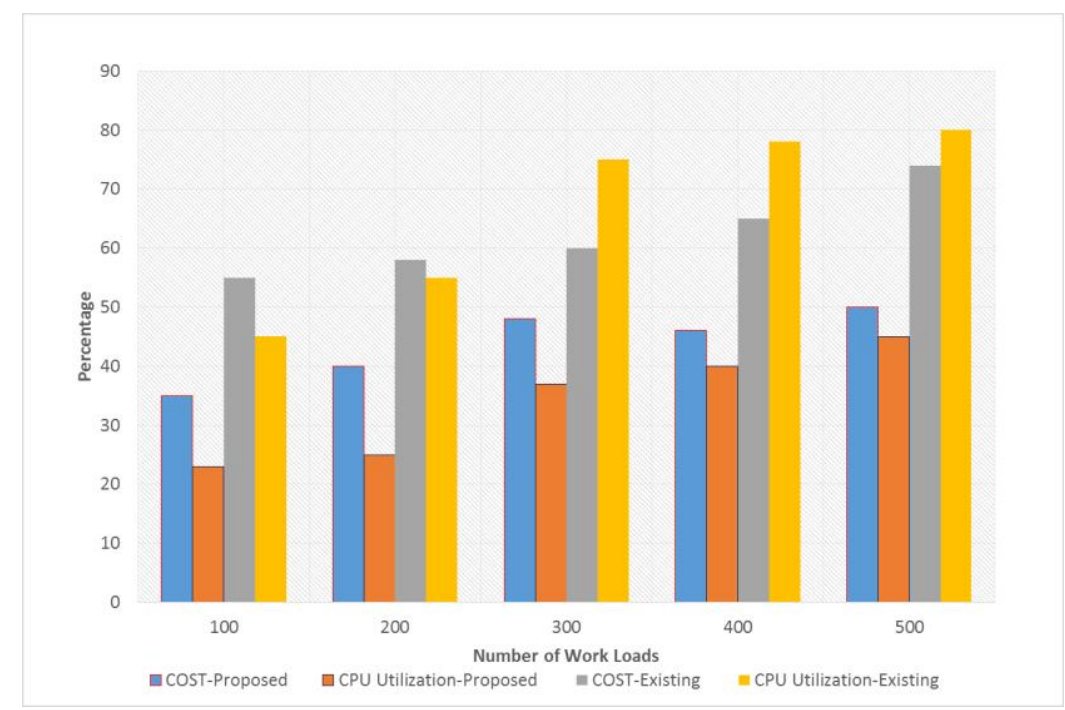

Figure.7 Cost and CPU Utilization

ISSN: 2582-418X (online) 
Journal of Information Technology and Digital World (2020)

Vol.02/ No. 01

Pages: 53-63

https://www.irojournals.com/itdw/

DOI: https://doi.org/10.36548/jitdw.2020.1.005

The figure. 8 represents the number of missed deadlines by the method put forth, the comparison with the existing method evinces the mechanism put forward performs better in migration by proper selection of virtual machine, based on the metric associated with the quality of service.

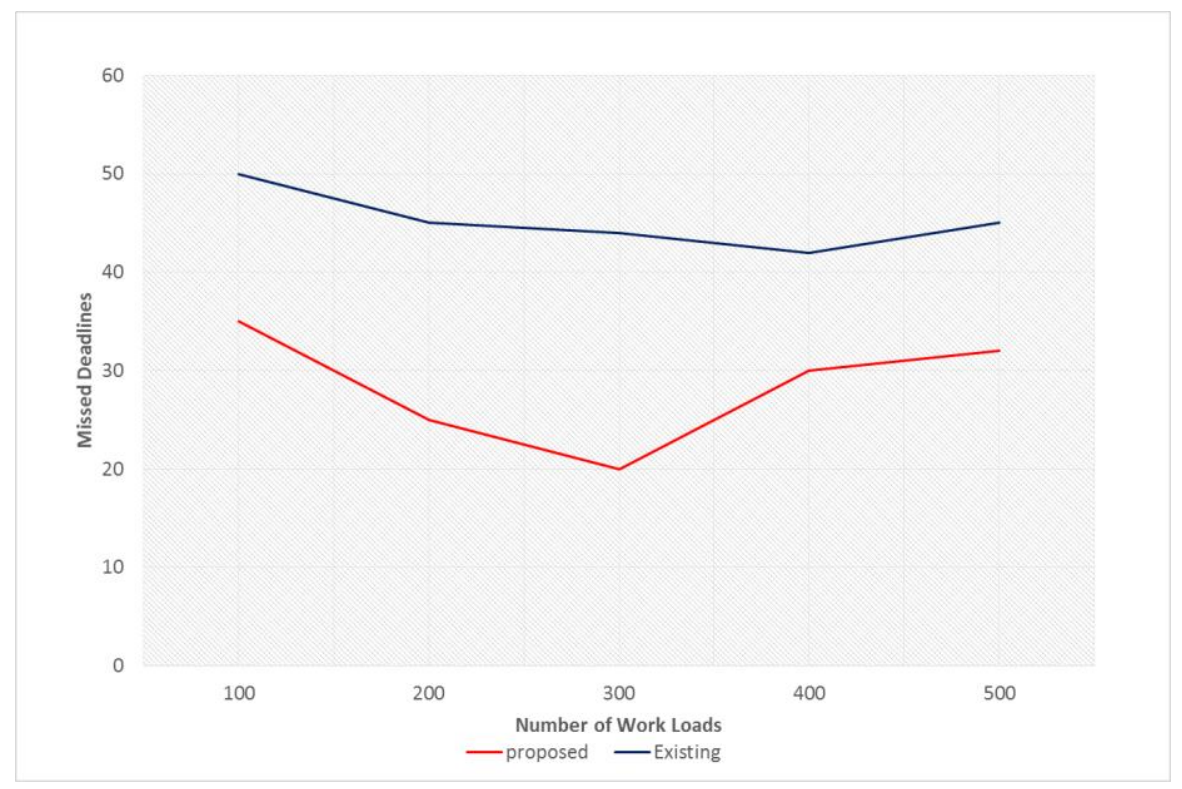

Figure.8 Missed Deadlines

\section{Conclusion}

The swift progress in the cloud service has paved way for the development multitudes of data centers of cloud, to handle the huge set of application adopted to it. so to handle to user requisitions with the assured services quality the paper has developed the migration scheme taking into consideration the multiple QoS metrics , such as the cost, latency, uptime, CPU utilization etc., with the help of the module in built identifies the appropriate VM with the satisfying metric and proceeds with the process of migration. The Evaluation results obtained evinces the performance of the proposed method against the existing. Further in future the paper aims to develop a selection algorithm based on machine learning to reduce the manual intervention in the process. 
Journal of Information Technology and Digital World (2020)

Vol.02/ No. 01

Pages: 53-63

https://www.irojournals.com/itdw/

DOI: https://doi.org/10.36548/jitdw.2020.1.005

\section{References}

[1] Zhang, Baoke, Karthikeyan Sabhanatarajan, Ann Gordon-Ross, and Alan George. "Real-time performance analysis of adaptive link rate." In 2008 33rd IEEE Conference on Local Computer Networks (LCN), pp. 282-288. IEEE, 2008.

[2] Gunaratne, Chamara, Kenneth Christensen, Bruce Nordman, and Stephen Suen. "Reducing the energy consumption of Ethernet with adaptive link rate (ALR)." IEEE Transactions on Computers 57, no. 4 (2008): 448-461.

[3] Beloglazov, Anton, Rajkumar Buyya, Young Choon Lee, and Albert Zomaya. "A taxonomy and survey of energy-efficient data centers and cloud computing systems." In Advances in computers, vol. 82, pp. 47-111. Elsevier, 2011.

[4] Galloway, Jeffrey M., Karl L. Smith, and Susan S. Vrbsky. "Power aware load balancing for cloud computing." In Proceedings of the World Congress on Engineering and Computer Science, vol. 1, pp. 19-21. 2011.

[5] Beloglazov, Anton, Jemal Abawajy, and Rajkumar Buyya. "Energy-aware resource allocation heuristics for efficient management of data centers for cloud computing." Future generation computer systems 28, no. 5 (2012): 755-768.

[6] Huang, Daochao, Dong Yang, Hongke Zhang, and Lei Wu. "Energy-aware virtual machine placement in data centers." In 2012 IEEE Global Communications Conference (GLOBECOM), pp. 3243-3249. IEEE, 2012.

[7] Maurya, Khushbu, and Richa Sinha. "Energy conscious dynamic provisioning of virtual machines using adaptive migration thresholds in cloud data center." International Journal of Computer Science and Mobile Computing 2, no. 3 (2013): 74-82.

[8] Graubner, Pablo, Matthias Schmidt, and Bernd Freisleben. "Energy-efficient virtual machine consolidation." It Professional 15, no. 2 (2012): 28-34.

[9] Farooqi, Abdul Majid. "Comparative Analysis of Green Cloud Computing." International Journal of Advanced Research in Computer Science 8, no. 2 (2017).

[10] Piraghaj, Sareh Fotuhi, Amir Vahid Dastjerdi, Rodrigo N. Calheiros, and Rajkumar Buyya. "A survey and taxonomy of energy efficient resource management techniques in platform as a service cloud." In Handbook of Research on End-to-End Cloud Computing Architecture Design, pp. 410-454. IGI Global, 2017.

[11] Kumar, Dinesh. "Review on task scheduling in ubiquitous clouds." J. ISMAC 1, no. 01 (2019): 72-80.

[12] Smys, S., \& Raj, J. S. (2019). A Stochastic Mobile Data Traffic Model for Vehicular Ad Hoc Networks. Journal of Ubiquitous Computing and Communication Technologies (UCCT), 1(01), 55-63.

[13] Bhalaji, N. (2019). Delay Diminished Efficient Task Scheduling and Allocation for Heterogeneous Cloud Environment. Journal of trends in Computer Science and Smart technology (TCSST), 1(01), 51-62. 
Journal of Information Technology and Digital World (2020)

Vol.02/ No. 01

Pages: 53-63

https://www.irojournals.com/itdw/

DOI: https://doi.org/10.36548/jitdw.2020.1.005

[14] .Shakya, S. (2019). An Efficient Security Framework for Data Migration in a Cloud Computing Environment. Journal of Artificial Intelligence, 1(01), 45-53.

[15] .Karunakaran, V. "A Stochastic Development of Cloud Computing Based Task Scheduling Algorithm." Journal of Soft Computing Paradigm (JSCP) 1, no. 01 (2019): 41-48.

[16] .Bashar, A. (2019). Secure And Cost Efficient Implementation Of The Mobile Computing Using Offloading Technique. Journal of Information Technology, 1(01), 48-57.

[17] Haladu, Mubarak, and Joshua Samual. "Optimizing Task Scheduling and Resource allocation in Cloud Data Center using Enhanced Min-Min Algorithm." IOSR J. Comput. Eng 18 (2016): $18-25$. 